\title{
Interventional closure vs. medical therapy of patent foramen ovale for secondary prevention of stroke: updated meta-analysis
}

\author{
Syed Raza Shah ${ }^{1}$
}

Received: 9 August 2018 / Accepted: 8 October 2018 / Published online: 29 October 2018

๑) Springer-Verlag GmbH Germany, part of Springer Nature 2018

Sirs:

We read with great interest the recent updated meta-analysis by Vukadinović $\mathrm{D}$ et al. [1] evaluating patent foramen ovale closure for prevention of cryptogenic stroke. The authors did a great work and we would like to congratulate them. However, we would like to comment on a few important issues in the study that are important for clinicians dealing with this situation and expand on the pooled analysis using the same data included in the current paper. The authors concluded that the interventional closure showed a marked benefit in preventing strokes with a paradoxical increased incidence of atrial fibrillation.

The same clinical question has been asked multiple times in the past. Excluding CLOSURE 1 data, which was the first study to answer this hypothetical question, the pooledeffect estimate hazard ratio for stroke prevention improved from 0.40 (95\% CI $\left.0.19-0.85 ; I^{2}=57.3\right)$ to 0.29 (95\% CI $\left.0.12-0.68 ; I^{2}=20.3\right)$. Additionally, for outcomes of stroke or transient ischemic attack (TIA), the authors calculated the events of stroke or TIA incorrectly, combining the numbers of patients undergoing stroke and the numbers of patients suffering TIA. Besides 1 participant being subjected to the same event more than once, the situation that both TIA and stroke may occur in the same individual participant was also involved. Furthermore, postprocedural atrial fibrillation needs to be considered in both the short and longer terms. Most studies report no difference in persistent atrial fibrillation. However, the increased short-term incidence of atrial fibrillation creates implications for anticoagulation in a patient who has already had a stroke.

Syed Raza Shah

syedraza91shah@live.com

1 North Florida Regional Medical Center, University of Central Florida (Gainesville), 6500 West Newberry Road, Gainesville, FL 32605, USA
Still, it is hard to choose a "close-all" strategy for patients with cryptogenic stroke. The authors rightly point out that a younger age is a good starting point because all trials include patients aged $<60$ years. This would suggest benefit of closure for young patients with a sizeable right-to-left shunt only. A patient-level meta-analysis to clarify this question will help clear the waters further on this issue. Furthermore, subgroup analysis according to gender, medical therapy (anticoagulation, anticoagulation plus antiplatelet, or antiplatelet), and shunt size should be addressed. No beneficial effects of patent foramen ovale closure were observed in patients with small or moderate shunt size or female patients. Additionally, benefit of patent foramen ovale closure for primary outcomes existed only by comparison with antiplatelet therapy, but not anticoagulation or anticoagulation plus antiplatelet therapy. While advances in technologies have helped elucidate many aspects of these diseases, many mysteries still remain. Therefore, an in-depth examination of cardiovascular outcome data in conjunction with basic science data is critical for a detailed understanding of benefits and risks of newer treatment modalities.

\section{Compliance with ethical standards}

Conflict of interest The author declares that there is no conflict of interest.

\section{Reference}

1. Vukadinović D, Schirmer SH, Vukadinović AN, Ukena C, Scheller B, Mahfoud F, Böhm M (2018) Interventional closure vs. medical therapy of patent foramen ovale for secondary prevention of stroke: updated meta-analysis. Clin Res Cardiol. https:// doi.org/10.1007/s00392-018-1334-z 